Draft VERSion March 21, 2022

Typeset using LATEX twocolumn style in AASTeX61

\title{
DENSITY FLUCTUATIONS IN THE SOLAR WIND DRIVEN BY ALFVÉN WAVE PARAMETRIC DECAY
}

\author{
Trevor A. Bowen, ${ }^{1}$ Samuel Badman,${ }^{1}$ Petr Hellinger,${ }^{2}$ and Stuart D. Bale ${ }^{1}$ \\ ${ }^{1}$ Physics Department and Space Sciences Laboratory, University of California, 7 Gauss Way, Berkeley, CA 94720, USA \\ ${ }^{2}$ Astronomical Institute, CAS, Bocni II/1401, CZ-14100 Prague, Czech Republic
}

Submitted to ApJL

\begin{abstract}
Measurements and simulations of inertial compressive turbulence in the solar wind are characterized by anticorrelated magnetic fluctuations parallel to the mean field and density structures. This signature has been interpreted as observational evidence for non-propagating pressure balanced structures (PBS), kinetic ion acoustic waves, as well as the MHD slow-mode. Given the high damping rates of parallel propagating compressive fluctuations, their ubiquity in satellite observations is surprising, and suggestive of a local driving process. One possible candidate for the generation of compressive fluctuations in the solar wind is Alfvén wave parametric instability. Here we test the parametric decay process as a source of compressive waves in the solar wind by comparing the collisionless damping rates of compressive fluctuations with growth rates of the parametric decay instability daughter waves. Our results suggest that generation of compressive waves through parametric decay is overdamped at $1 \mathrm{AU}$, but that the presence of slow-mode like density fluctuations is correlated with the parametric decay of Alfvén waves.
\end{abstract}

Keywords: instabilities — plasmas — solar wind 


\section{INTRODUCTION}

Observations of the solar wind contain a characteristic anti-correlation between density fluctuations and magnetic perturbations parallel to the mean field. Tu \& Marsch (1994) interpreted observed correlations on hourly timescales between magnetic pressure, thermal pressure, and density fluctuations from Helios as nonpropagating pressure balance structures (PBS). Using spacecraft potential measurements as a proxy for density fluctuations, Kellogg \& Horbury (2005) reported the same anti-correlation in Cluster data, noting that PBS are consistent with the perpendicular limit of the ionacoustic wave. Yao et al. (2011), using wavelet coherence analysis, demonstrated this anti-correlation through the range of inertial scales; subsequent work interpreted these observations as PBS driven by either slow-modes or mirror modes arising from temperature anisotropies (Yao et al. 2013a,b). Through statistical analysis of a decade of Wind observations, Howes et al. (2012) attributed the compressible component of magnetic fluctuations entirely to the kinetic slow-mode. However, comparisons between a decade of Wind observations and numerical predictions made in both kinetic and MHD simulations by Verscharen et al. (2017) suggest that the MHD slow-mode best explains the compressible component of the solar wind.

Strong collisionless Landau damping of kinetic slowmode waves propagating parallel to the magnetic field is expected for solar wind parameters at $1 \mathrm{AU}$ (Barnes 1966). For this reason, the generation, presence, and damping of kinetic slow-mode fluctuations in the solar wind remains an open question in space physics. Klein et al. (2012) argued that the distribution of slowmode waves in the solar wind is consistent with critically balanced turbulence, in which non-linear interactions between Alfvénic and slow-mode fluctuations cascade weakly damped perpendicular compressible fluctuations at large scales to smaller parallel scales. The damping of these waves could provide a source of heating in the solar wind (Narita \& Marsch 2015).

In this letter, we explore the parametric decay instability (PDI) as a process for generating compressive fluctuations in the solar wind. In MHD, the PDI is recognized as the instability of circularly polarized Alfvén eigenmodes at high fluctuation amplitudes (Derby 1978; Goldstein 1978). For low $\beta$ plasmas, the large amplitude Alfvénic fluctuation (mother wave) couples to two daughter waves: a parallel propagating compressive fluctuation, and a backwards propagating Alfvén wave. A class of parametric instabilities exist outside the low $\beta$ PDI decay: e.g. at $\beta>1$ the instability is dominated by the growth of forward propagating Alfvénic daugh- ter waves at twice the mother wave frequency (Jayanti \& Hollweg 1993; Hollweg 1994).

Simulations of high amplitude circularly polarized Alfvén waves have verified the PDI process in the MHD regime, and have suggested that PDI daughter waves can seed turbulent cascades Del Zanna et al. (2001); Tenerani \& Velli (2013); Malara et al. (2001). Simulations have extended the PDI to large amplitude Alfvén waves with arc and linear polarizations, nonmonochromatic wave distributions, and oblique propagation Del Zanna (2001); Matteini et al. (2010); Del Zanna et al. (2015). Furthermore, kinetic simulations recover PDI in multi-species models, including effects such as, the preferential heating of $\alpha$ particles, proton core heating, and beam formation Araneda et al. (2008); Araneda et al. (2009); Kauffmann \& Araneda (2008). Numerical simulations of PDI in a turbulent solar wind demonstrate the generation of slow-mode fluctuations from the decay of Alfvénic fluctuations in a relatively robust theoretical scenario Shi et al. (2017).

\section{DATA}

We use 10 years of solar wind measurements at $1 \mathrm{AU}$ from the NASA Wind mission ranging from 1996 January 1 through 2005 December 31. Data from the Magnetic Field Investigation (MFI) Lepping et al. (1995), Solar Wind Experiment (SWE) Ogilvie et al. (1995), and Three Dimensional Plasma (3DP) experiment Lin et al. (1995) are separated into non-overlapping 300 second intervals. Intervals are excluded when Wind's geocentric distance is less than $35 R_{E}$, the average solar wind speed is $<250 \mathrm{~km} / \mathrm{s}$, or if an interval is missing $>15 \%$ of coverage from any instrument. Data gaps are interpolated when $<15 \%$ of measurements are missing. Additionally, intervals are excluded if there is significant discrepancy between 3DP and SWE measurements of mean proton density such that $\left|\left(n_{S W E}-n_{3 D P}\right)\right| / n_{S W E}>0.1$. The resulting data set consists of 533222 individual 300 second intervals. Techniques from Pulupa et al. (2014) were used to obtain electron densities and temperatures for 282286 of these intervals.

The 3 s cadence 3DP "on board" proton moment measurements are interpolated to the MFI time base. For each interval, measurements of density, velocity, and magnetic field (represented as $n, \mathbf{v}$, and $\mathbf{B}$ ) are separated into mean and fluctuation quantities using time averaged quantities, denoted as $\langle\ldots\rangle$. For example, the mean magnetic field, $\mathbf{B}_{\mathbf{0}}$, is determined through $\langle\mathbf{B}\rangle=\mathbf{B}_{\mathbf{0}}$ while the fluctuation field is determined through $\mathbf{B}=\mathbf{B}_{\mathbf{0}}+\delta \mathbf{B}$. Vector fluctuations are rotated into a field aligned coordinate $(\mathrm{FAC})$ system, $\delta \mathbf{B}_{F A C}=$ 
$\left(\delta B_{\perp 1}, \delta B_{\perp 2}, \delta B_{\|}\right)$, with the parallel fluctuations, $\delta B_{\|}$, defined along the mean field direction $\mathbf{B}_{\mathbf{0}} /\left|B_{0}\right|$.

Each interval is characterized through root-meansquare (RMS) quantities normalized to the mean value

$$
\overline{\delta n}=\sqrt{\left\langle\delta n^{2}\right\rangle} / n_{0} .
$$

Vector fluctuations characterizing each interval separated into parallel and perpendicular RMS values of the form

$$
\delta \bar{B}_{\perp}=\frac{\sqrt{\left\langle\delta B_{\perp}^{2}\right\rangle}}{B_{0}}=\frac{\sqrt{\left\langle\delta B_{\perp 1}^{2}+\delta B_{\perp 2}^{2}\right\rangle}}{B_{0}}
$$

and

$$
\delta \bar{B}_{\|}=\frac{\sqrt{\left\langle\delta B_{\|}^{2}\right\rangle}}{B_{0}} .
$$

The FAC fluctuation quantities are used in deriving Elsässer variables,

$$
\mathbf{z}^{ \pm}=\delta \mathbf{v}_{F A C} \pm \delta \mathbf{b}_{F A C}
$$

normalized cross helicity,

$$
H_{c}=\frac{\left\langle\delta \mathbf{b}_{F A C} \cdot \delta \mathbf{v}_{F A C}\right\rangle}{\left\langle\delta b^{2}\right\rangle+\left\langle\delta v^{2}\right\rangle} ;
$$

and the zero lag cross correlation between parallel field fluctuations and density,

$$
C\left(\delta n, \delta B_{\|}\right)=\frac{\sum_{i=0}^{N-1} \delta n_{i} \delta B_{\| i}}{\sqrt{\sum_{i=0}^{N-1} \delta n_{i}^{2}} \sqrt{\sum_{i=0}^{N-1} \delta B_{\| i}^{2}}} .
$$

The magnetic field has been normalized as $\delta \mathbf{b}=$ $\delta \mathbf{B} / \sqrt{\mu_{0} n_{p} m_{p}}$.

\section{MODEL PROPAGATION DIRECTION}

For slow-mode waves with propagation vector, $\mathbf{k}$, such that $\mathbf{k}=\left(k_{\perp 1}, 0, k_{\|}\right)$it follows that $\delta \mathbf{v}=\left(\delta v_{\perp 1}, 0, \delta v_{\|}\right)$. From the MHD induction equation $\omega \delta \mathbf{B}=(\mathbf{k} \cdot \delta \mathbf{v}) \mathbf{B}_{\mathbf{0}}-$ $\left(\mathbf{k} \cdot \mathbf{B}_{\mathbf{0}}\right) \delta \mathbf{v}$, it is derived that

$$
\tan \theta_{s m}=\left|\frac{k_{\perp}}{k_{\|}}\right|=\left|\frac{\delta B_{\|}}{\delta B_{\perp}}\right|,
$$

where $\theta_{s m}$ gives the slow-mode propagation angle relative to the mean magnetic field. The limit of small $\delta B_{\|}$, corresponds to quasi-parallel propagation of the slowmod; for exact parallel propagation, $\delta B_{\|}=0$, the compressive component of the slow-mode becomes purely acoustic.

Figure 1 (top) shows joint probability distributions of $\left|H_{c}\right|$ and $\theta_{s m}$ normalized to the maximum counts in each column. For a shear Alfvén wave $H_{c}= \pm 1$; our observations show that, for small propagation angles, the majority of intervals have a cross-helicity similar to shear Alfvén waves.

Figure 1 (middle) shows the column normalized joint distribution of $\theta_{s m}$ and the ratio of Elsässer energies: $e^{+} / e^{-}$, where $e^{ \pm}=\left\langle\left(z^{ \pm}\right)^{2}\right\rangle$. Intervals consisting of pure outward propagating Alfvén waves have large Elsässer ratios: i.e. intervals with $z^{+} \gg z^{-}$correspond to $e^{+} \gg e^{-}$. Intervals containing balanced compositions of $z^{-}$and $z^{+}$fluctuations have Elsässer ratios $\sim 1$. We find that intervals with small propagation angles are dominated by $z^{+}$fluctuations. Intervals with larger $\theta_{s m}$ show balanced $z^{+}$and $z^{-}$energies, suggesting that these intervals are not easily represented as single wave fluctuations, but must be modeled by either counterpropagating Alfénic fluctuations or a quasi-linear superposition of wave-modes (Klein et al. 2012).

Figure 1 (bottom) shows the the column normalized joint distribution of $C\left(\delta n, \delta B_{\|}\right)$and $\theta_{s m}$. The transition to highly anti-correlated density $\delta n$ and parallel magnetic fluctuations $\delta B_{\|}$occurs at approximately the same propagation angle, $\theta_{s m} \sim 20^{\circ}$, as cross helicity decreases and the Elsässer energies balance.

Two regimes are apparent: the first consists of intervals characterized by large cross helicity and 'outward' propagating energies. The second regime consists of highly compressible intervals with mixed cross helicity, balanced Elsässer energies, and strong density-field correlations consistent with slow-mode fluctuations. Howes et al. (2012) restricted analysis to intervals with $\delta n>0.5$ $\mathrm{cm}^{-3}$, arguing that lower density intervals may be subject to instrumental noise; the uniform and simultaneous transitions in $H_{c}, e^{+} / e^{-}$, and $C\left(\delta n, \delta B_{\|}\right)$with $\theta_{s m}$ without restricting density amplitudes suggests that our analysis is insensitive to noise due to low amplitude densities.

\section{DAMPING}

Historically, the presence of kinetic slow-mode fluctuations in the solar wind has been questioned due to the heavy collisionless damping expected for solar wind parameters at 1 AU. (Barnes 1966). However, Figure 1 (bottom) reveals the presence of compressive fluctuations with quasi-parallel propagation (e.g. $\theta_{s m} \sim 20^{\circ}$ ). Verscharen et al. (2017) previously argued that observations of the compressive solar wind plasma are best represented as oblique, weakly damped, MHD slow-mode waves which propagate more freely through the heliosphere; we adopt their expression for ion acoustic (IA) damping rates derived for Maxwellian particle distributions:

$$
\gamma_{I A} / \omega_{s} \simeq-\sqrt{\pi} \frac{c_{s}^{3}}{w_{\| p}^{3}} \frac{e^{-c_{s}^{2} / w_{\| p}^{2}}}{1+3 w_{\| p}^{2} / c_{s}^{2}},
$$


Normalized Counts
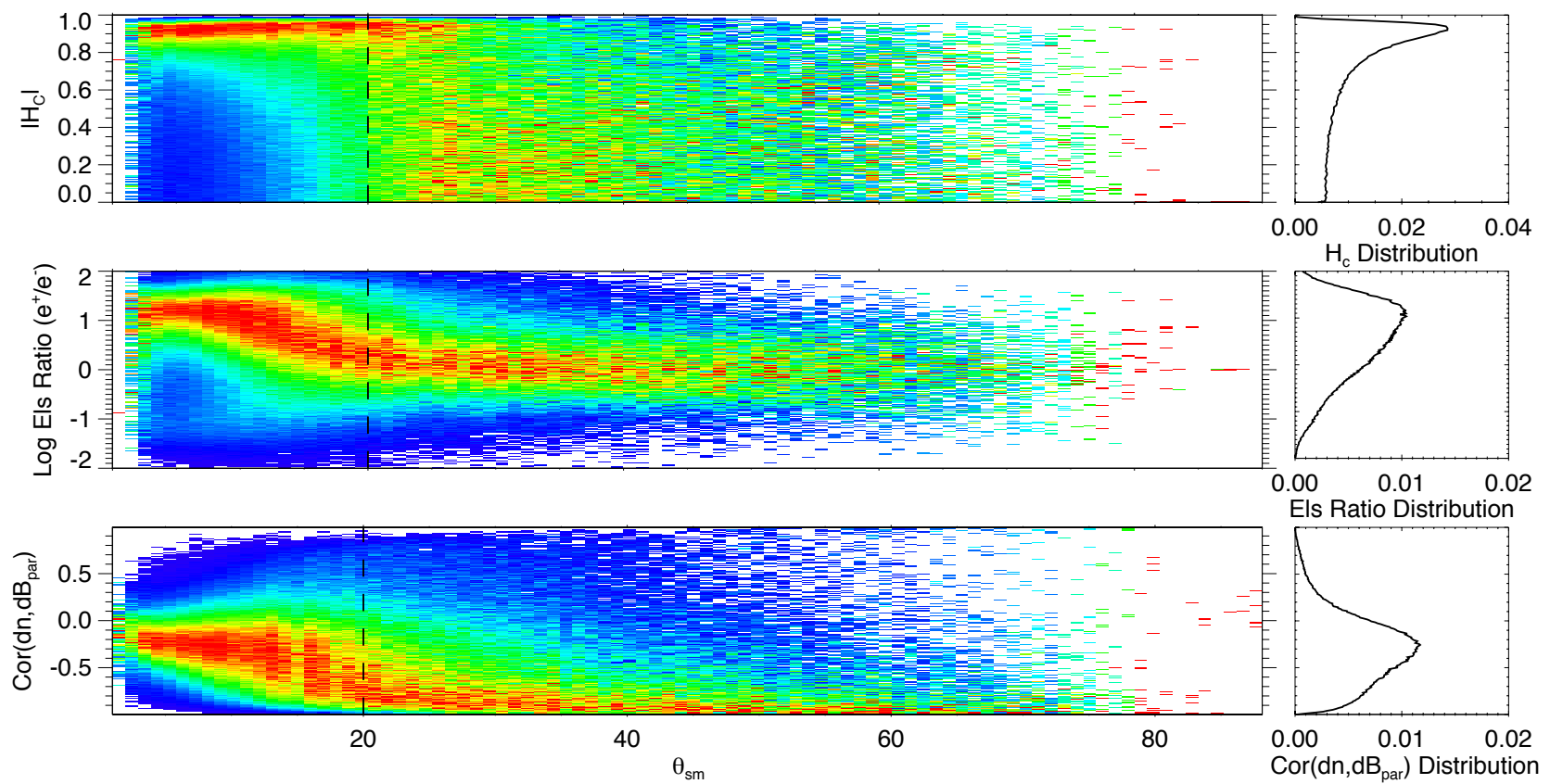

Figure 1. (Top) Joint distribution of normalized cross helicity, $H_{c}$ and slow-mode propagation angle $\theta_{s m}$. Data are column normalized within each angle bin $\left(1^{\circ}\right.$ resolution). The distribution of $H_{c}$ observations shown on the right. (Middle) Column normalized distribution of the Elsässer ratio and $\theta_{s m}$. The distribution of Elsässer ratios is plotted to the right. (Bottom) Joint distribution of $C\left(\delta n, \delta B_{\|}\right)$, and the slow-mode propagation angle, $\theta_{s m}$. The data are column normalized and the distribution of $C\left(\delta n, \delta B_{\|}\right)$observations plotted to the right. A dashed line identifies a transition observed in all three panels at $\theta_{s m} \sim 20^{\circ}$.

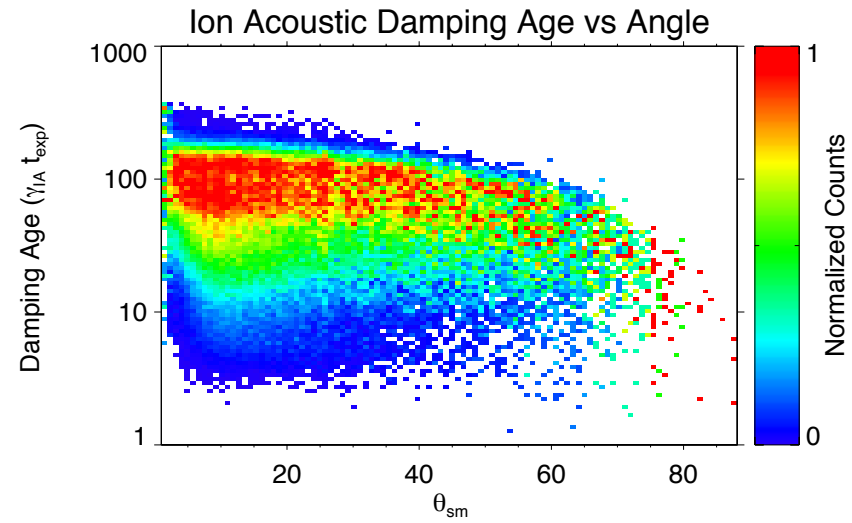

Figure 2. Joint distribution of expansion normalized ionacoustic damping rates, $\gamma_{I A} t_{\exp },\left(t_{\exp }=1 \mathrm{AU} / V_{s w}\right)$ and slow-mode propagation angle $\theta_{s m}$. Data are restricted to intervals with fitted electron temperatures. The distribution is column normalized in each angle bin $\left(1^{\circ}\right.$ resolution). Damping ages, $\gamma_{I A} t_{e x p}$, are uniformly greater than unity.

where the ion acoustic frequency is $\omega_{s}=k_{\|} c_{s}$, the parallel thermal proton speed is $w_{\| p}=\sqrt{2 k_{B} T_{\| p} / m_{p}}$, the ion-acoustic speed is

$$
c_{s}=\sqrt{\frac{3 k_{B} T_{\| p}+k_{B} T_{\| e}}{m_{p}}},
$$

$k_{\|}=k \cos \theta_{s m}, m_{p}$ is the proton mass, and $T_{\| j}$ is the parallel temperature for species $j$.

Figure 2 shows IA damping rates normalized to the solar wind expansion time $t_{\text {exp }}=1 \mathrm{AU} / V_{s w}$ as a function of $\theta_{s m}$. Data are restricted to intervals with fits of electron distributions (Pulupa et al. 2014). The majority of expansion normalized damping ages are above 10, and uniformly above unity, suggesting that even highly oblique propagating kinetic slow-mode like fluctuations undergo significant damping over $1 \mathrm{AU}$ propagation.

Figure 3 (top) shows the column normalized joint distribution of $\overline{\delta n}$ and $C\left(\delta n, \delta B_{\|}\right)$while Figure 3 (bottom) shows that the slow-mode correlation emerges even for quasi-parallel propagation $\left(\theta_{s m}<20^{\circ}\right)$. If these fluctuations are associated with a strongly damped kinetic slow-mode, then their presence at $1 \mathrm{AU}$ demands a local driving process. 


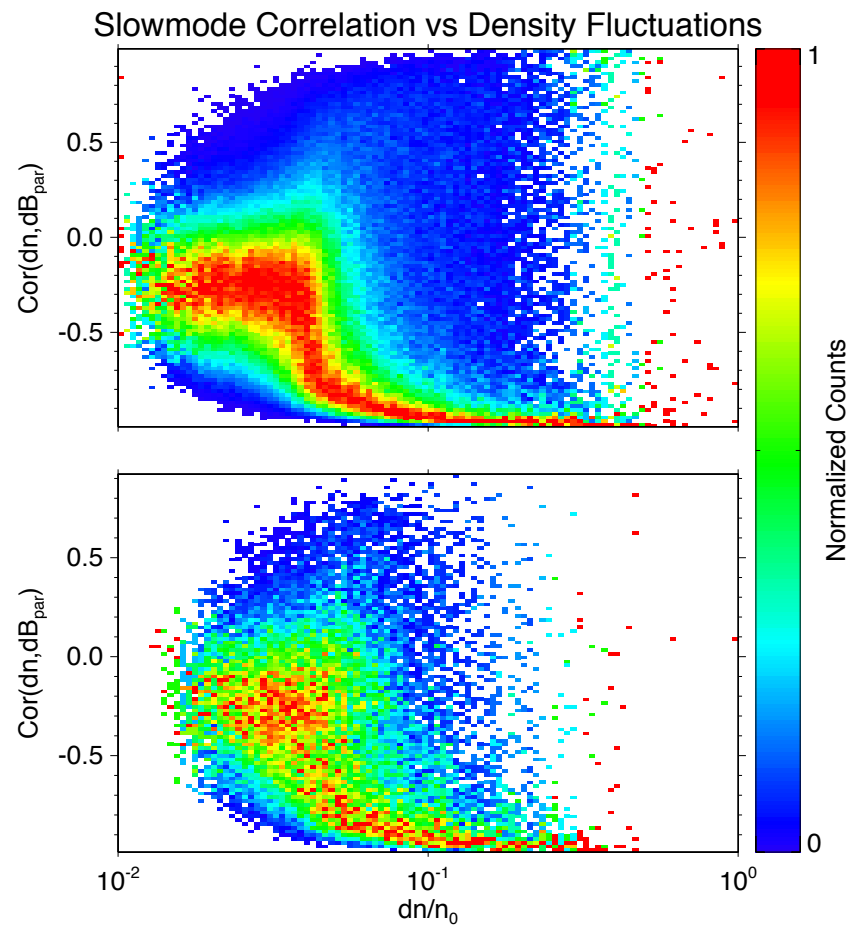

Figure 3. (Top) Joint probability distribution of densityparallel magnetic fluctuation cross-correlation, $C\left(\delta n, \delta B_{\|}\right)$, and density fluctuation amplitude, $\overline{\delta n}$. (Bottom) Restricting data to intervals with $\beta<0.5$ and $\theta_{\text {sm }}<20^{\circ}$ reveals the presence of fluctuations with slow-mode like density-field correlations propagating quasi-parallel to the mean magnetic field. Data are column normalized to the maximum counts of $C\left(\delta n, \delta B_{\|}\right)$in each $\overline{\delta n}$ bin.

\section{PARAMETRIC DECAY}

To test whether parametric coupling between wave modes generate compressive fluctuations from the decay of large amplitude Alfvénic fluctuations we compare our observations with analytically derived parametric growth rates. Following Derby (1978) and Goldstein (1978), a dispersion relation for the evolution of a compressive wave coupled to an MHD mother wave is given by:

$$
\begin{aligned}
(\omega+k+2) & (\omega+k-2)(\omega-k)\left(\omega^{2}-\beta k^{2}\right) \\
& -\left(\frac{\delta B_{\perp}}{B_{0}}\right)^{2} k^{2}\left(\omega^{3}+k \omega^{2}-3 \omega+k\right)=0 .
\end{aligned}
$$

This $5^{\text {th }}$ order polynomial, with $k$ and $\omega$ corresponding to wave number and frequency of the compressive daughter wave normalized to mother wave quantities, depends only on $\beta$ and $\delta B_{\perp} / B_{0}$. There is a small range of $k_{L}<k<k_{U}$ for which a single conjugate pair of complex solutions exist. The imaginary part of the solution, i.e. the parametric decay rate $\gamma_{p d}$, corresponding to the fastest growing decay product is determined numerically given observed values of $\beta$ and $\delta \bar{B}_{\perp}$.

Figure 4 (left) shows the joint distribution of $\delta \bar{B}_{\perp}$ and $\beta$. Contours of numerically determined $\gamma_{p d} / \omega_{0}$, plotted as solid black lines, bound the data at $\gamma_{p d} / \omega_{0} \sim$ $0.05-0.1$, suggesting that the solar wind at $1 \mathrm{AU}$ is bounded by PDI growth. Figure 4 (right) shows the same distribution of $\delta \bar{B}_{\perp}$ and $\beta$ with the average value of $\overline{\delta n}$ projected onto the plane. The median $\overline{\delta n}$ is 0.034 , ranging between $[0.01,1.10]$. Our results show that intervals with the largest density fluctuations correspond to intervals of high parametric growth rates.

Two sets of contours are overlaid in Figure 4 (right). The first set, $\gamma_{p d} t_{\text {exp }}$ plotted in red, correspond to PDI growth rates normalized to solar wind expansion time. Normalizing by expansion timescale requires estimating $\gamma_{p d}$, with physical units of $\mathrm{s}^{-1}$, from contours of $\gamma_{p d} / \omega_{0}$. Using

$$
\omega_{0}=k_{\|} V_{a l f},
$$

where $k$ is given by the Taylor hypotheses, $k=1 /\left(\tau V_{s w}\right)$, where $\tau$ is the interval duration $(300 \mathrm{~s})$, and $k_{\|}=k \cos \theta$, it follows that,

$$
\gamma_{p d} t_{e x p}=\frac{\gamma_{p d}}{\omega_{0}} \frac{1 \mathrm{AU}}{\tau} \frac{V_{a l f}}{V_{s w}^{2}} \cos \theta
$$

Contours of $\gamma_{p d} / \omega_{0}$, are normalized using mean values of $V_{s w}$ and $V_{a l f}$ in each bin of the joint $\delta \bar{B}_{\perp}$ and $\beta$ distribution. We choose an oblique propagation angle of $\theta=80^{\circ}$ in accordance with observations of anisotropy in the solar wind (Horbury et al. 2008). At oblique propagation angles $\cos \theta$ varies weakly with $\theta$, implying that our results are robust to specific oblique angle chosen. Previous work by Matteini et al. (2010) and Del Zanna et al. (2001) has shown that the decay of oblique waves is analogous to parallel decay, scaling as $\cos \theta$; furthermore, Chandran (2017) demonstrate that non-linear PDI interactions for oblique waves occur between parallel components, generating an inverse parallel cascade of Alfvén waves.

Figure 4 (right) shows that intervals undergoing a single iteration of parametric decay demonstrate enhanced density fluctuations, suggesting that density fluctuations are correlated with growing compressive PDI daughter waves. Though parametric decay rates measured at Wind are fairly small, propagation over $1 \mathrm{AU}$ allows for growth of the instability. Additionally, contours of $\gamma_{p d} t_{\text {exp }}$ show that PDI growth could be smaller than our theoretical rates for circularly polarized, parallel propagating waves, and still generate compressive fluctuations at $1 \mathrm{AU}$. 


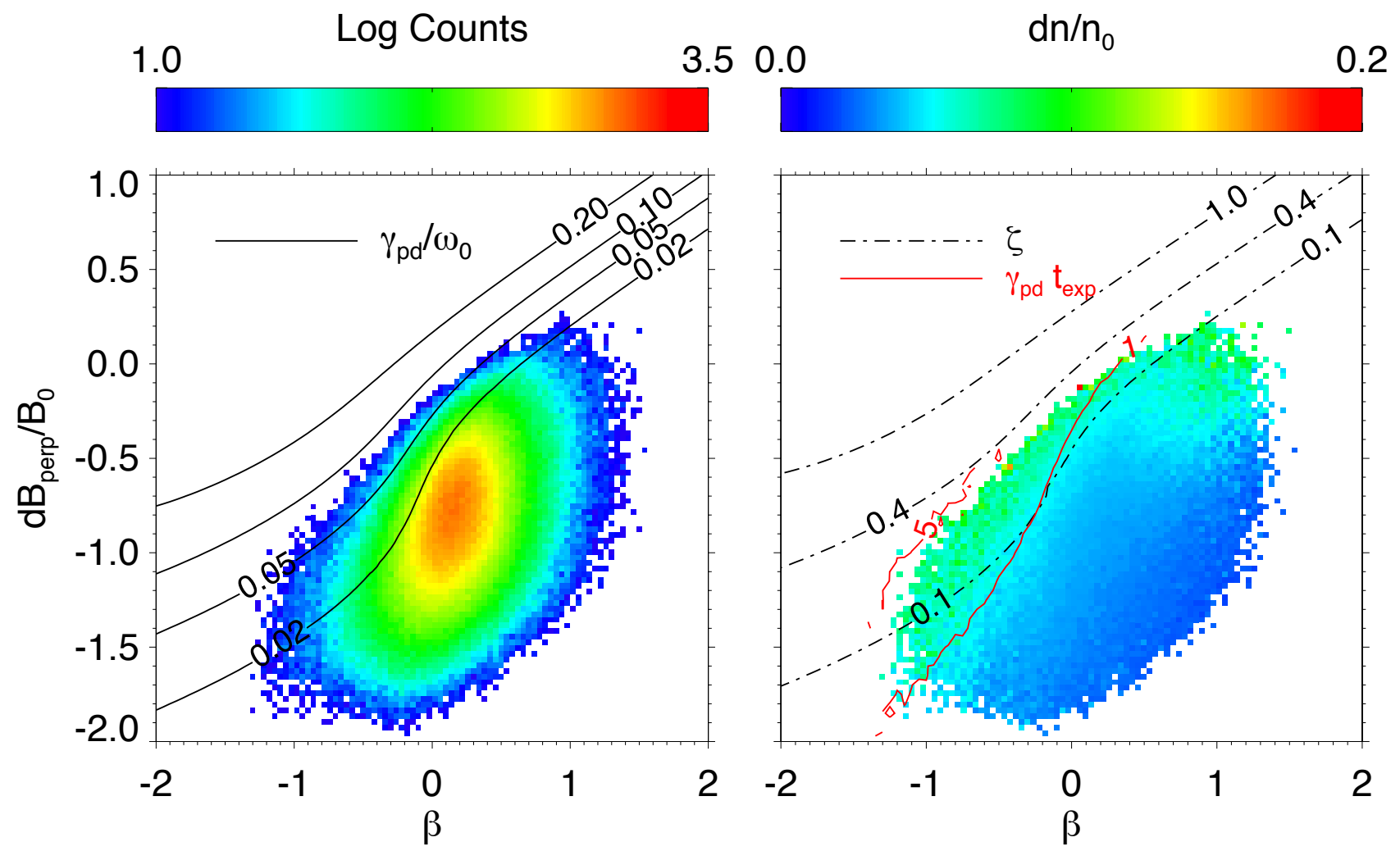

Figure 4. (Left) Joint distribution of $\delta \bar{B}_{\perp}$ and $\beta$. Contours of the parametric growth rate normalized to the linear wave frequency are shown in solid black. Data are bounded by high PDI growth rates. (Right) Joint distribution of $\delta \bar{B}_{\perp}$ and $\beta$ with color scale given by mean values of $\overline{\delta n}$. Contours of the expansion normalized parametric decay rate, $\gamma_{p d} t_{\text {exp }}$ shown in red, suggest that several iterations of the decay may occur over 1 AU propagation. Contours of $\zeta=\frac{\gamma_{p d} / \omega_{0}}{\gamma_{I A} / \omega_{s}}$ are shown in dashed black lines. Though large density fluctuations correspond to the largest values of $\zeta$, generation of density fluctuations through PDI is overdamped. 
Uncertainty in contours of $\gamma_{p d} t_{\text {exp }}$ are determined through the fractional error of Equation 3, derived as

$$
\begin{aligned}
& \frac{\Delta\left(\gamma_{p d} t_{\text {exp }}\right)}{\gamma_{p d} t_{\text {exp }}}= \\
& \sqrt{\left(4 \frac{\Delta V_{s w}}{<V_{s w}>}\right)^{2}+\left(\frac{\Delta V_{a l f}}{<V_{a l f}>}\right)^{2}-4 \frac{\Delta V_{s w} \Delta V_{a l f}}{V_{s w} V_{a l f}}}
\end{aligned}
$$

The ensemble mean values follow as $\left\langle V_{s w}\right\rangle=453$ $\mathrm{km} \mathrm{s}^{-1}$ and $\left\langle V_{\text {alf }}\right\rangle=60 \mathrm{~km} \mathrm{~s}^{-1}$, with standard deviations of $\Delta V_{s w}=108 \mathrm{~km} \mathrm{~s}^{-1}$ and $\Delta V_{a l f}=34 \mathrm{~km} \mathrm{~s}^{-1}$ respectively. Measurements of $V_{\text {alf }}$ and $V_{s w}$ are correlated with a value of $\rho=0.38$. Evaluating $\Delta\left(\gamma_{p d} t_{\text {exp }}\right) / \gamma_{p d} t_{\text {exp }}$ gives fractional uncertainty on the contours of $60 \%$. For a single instance of the parametric decay, $\gamma_{p d} t_{e x p}=1$, uncertainty of $60 \%$ does not significantly impact the contour location.

The second set of contours in Figure 4 (right), $\zeta=$ $\frac{\gamma_{p d} / \omega_{0}}{\gamma_{I A} / \omega_{s}}$, represent the ratio of PDI growth rates to IA damping rates. High values of $\zeta$ presumably indicate the presence of driven compressive daughter waves. Intervals with low values of $\zeta$ should dissipate compressive fluctuations faster than the PDI can drive them. Contours of $\zeta$ are constructed by normalizing the contours of $\gamma_{p d} / \omega_{0}$ to the observed median dimensionless damping rates, median $\left(\gamma_{I A} / \omega_{s}\right)$. For intervals with available electron fit data and $\beta<0.5$ the median value of $\gamma_{I A} / \omega_{s}$ is 0.26 , with a standard deviation of $\sim 0.05$. This leads to a fractional error to the contours of $\Delta \zeta / \zeta \sim 15 \%$. Figure 4 (right) shows that though intervals with large density fluctuations occur with greater values of $\zeta$, compressive fluctuations driven by parametric decay are overdamped, i.e. have PDI growth rates less than IA damping rates.

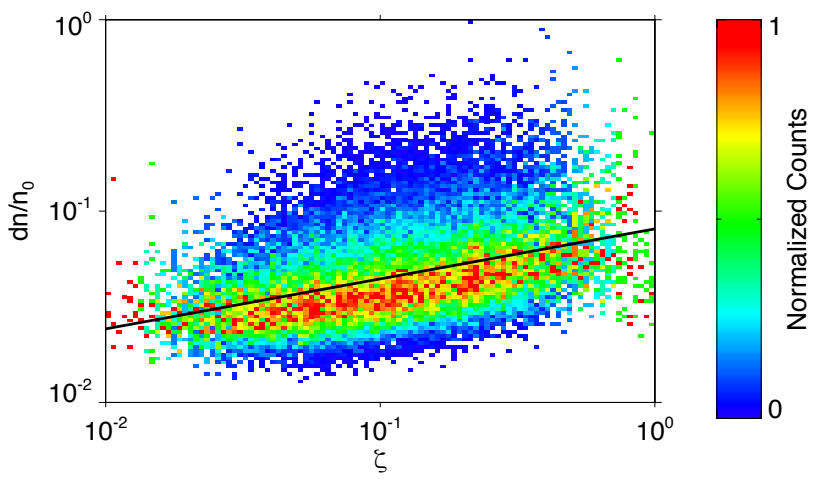

Figure 5. Distribution of $\overline{\delta n}$ and $\zeta$. Data are column normalized to each $\zeta$ bin. A least squares fit to the data, shown as a black line, gives a power law of 0.25 and a Pearson correlation of 0.36
Figure 5 shows the scaling of $\overline{\delta n}$ with $\zeta$ computed for each interval. Data are restricted to $\beta<0.5$, where PDI generates compressive daughter waves. A positive correlation is observed between the density fluctuations and $\zeta$. A least squares fit in logarithmic space suggests a power law relationship of $\overline{\delta n} \sim(\zeta)^{0.26}$ with a Pearson cross correlation of $\rho=0.36$; again we note that approximately $99 \%$ of the data are overdamped. When a least square fit is performed between $\overline{\delta n}$ and the dimensionless parametric growth rate, $\gamma_{p d} / \omega_{0}$ we recover a power law relation of $\overline{\delta n} \sim\left(\gamma_{p d} / \omega_{0}\right)^{0.26}$ and a Pearson correlation of $\rho=0.37$. Suggesting that density fluctuations $\overline{\delta n}$ are weekly dependent on the IA damping rate. Additionally, the positive correlation between parametric instability growth rate and density fluctuations in the solar wind suggests that compressive fluctuations may be related to PDI. Both of these correlations are stronger than the correlation between amplitudes of $\delta \bar{B}_{\perp}$ and $\overline{\delta n}$, for which we find $\rho=0.24$.

\section{DISCUSSION}

Our results show that the compressive component of solar wind plasma is not restricted to fluctuations propagating perpendicular to the magnetic field. If these compressive fluctuations correspond to a kinetic slowmode, their presence likely requires a driving process. Previous work has invoked a scalar turbulent cascade to explain the replenishing of slow-mode like fluctuations (Klein et al. 2012; Chen et al. 2012).

In this letter, we suggest that parametric coupling of large amplitude Alfvén waves to compressional modes may exist in the solar wind. We consider parametric decay as a low $\beta$ parallel process; however, the decay of oblique shear Alfvén waves generates a spectrum of fluctuations over a range of angles relative to the mean magnetic field (Matteini et al. 2010). One complication is that the parametric growth rates adopted from Derby (1978) are relevant for low $\beta$ plasmas; however, we note that Tenerani et al. (2017) demonstrate that pressure anisotropies allow PDI growth at higher $\beta$.

Parametric decay has been verified in analytic and computational studies but it has yet to be observed in the solar wind. Parametric coupling is an attractive physical mechanism for explaining compressive fluctuations in the solar wind for two reasons. First, backwards propagating Alfvénic fluctuations, a product of PDI, are required for non-linear interactions leading to critically balanced turbulence (Tenerani \& Velli 2013; Malara et al. 2001). Second, parametric coupling preferentially excites slow-mode fluctuations; which may explain the distribution of fluctuations observed at $1 \mathrm{AU}$ : 
Alfvénic $\sim 90 \%$, slow-mode $\sim 10 \%$, and very little fastmode contribution (Klein et al. 2012).

The results presented in Figure 1, in which slowmode fluctuations are observed simultaneously with balanced (i.e. equally distributed backwards and forwards propagating Alfvénic fluctuations) intervals provide compelling observational evidence for parametric decay. Furthermore, observations from Wind show that high PDI growth rates bound the distribution of data, suggesting that parametric coupling may limit the amplitude of Alfvénic fluctuations. The results in Figure 4 show that intervals of solar wind which undergo a single growth of parametric decay have enhanced density fluctuations.

Though the IA damping term given in Equation 1 is greater than PDI growth rates, we note that nonMaxwellian particle distributions in the solar wind may significantly lower the IA damping rates. In particular, we highlight the results of Verscharen et al. (2017), which suggest that slow-mode fluctuations in the solar wind are fluid like with low damping rates. Reducing IA damping by approximately a factor of 5 would provide underdamped growth of compressive fluctuations through PDI.

Additionally, scaling arguments give much higher PDI rates in the inner heliosphere, increasing generation of compressive waves through PDI near the sun (Tenerani \& Velli 2013). The launch of the NASA Parker Solar Probe mission in July 2018 will reveal whether parametric coupling between fluctuations play an increased role in the inner heliosphere.
In considering the growth rate of the PDI relative to the linear wave time, $\omega_{0}$, we do not take into account the effect of turbulence solar wind. The non-linear interaction time between Alfvénic fluctuations likely plays an important role in parametric coupling. Specifically, it is likely that a critically balanced cascade with nonlinear interactions occuring on order the wave propagation time could disrupt parametric coupling between Alfvénic and slow-mode like fluctuations Schekochihin et al. (2009). This idea has been touched upon in Shi et al. (2017), in which reduced PDI growth rates were found in a turbulent plasma. Our future work looks to address the physics of parametric coupling in a turbulent plasma and to compare the growth of compressive modes through PDI versus their recycling through a turbulent cascade.

The authors would like to acknowledge helpful discussions with Marco Velli, Anna Tenerani, and Alfred Mallet. We would additionally like to extend our thanks to Marc Pulupa and Chadi Salem for providing processed electron data from Wind/3DP. T.A.B. is supported by NASA Earth and Space Science Fellowship NNX16AT22H. P.H. acknowledges grant 18-08861S of the Czech Science Foundation. Wind/3DP data analysis at UC Berkeley is supported in part by NASA grant NNX16AP95G.

\section{REFERENCES}

Araneda, J. A., Maneva, Y., \& Marsch, E. 2009, Physical

Review Letters, 102, 175001

Araneda, J. A., Marsch, E., \& F.-Viñas, A. 2008, Phys.

Rev. Lett., 100, 125003

Barnes, A. 1966, Physics of Fluids, 9, 1483

Chandran, B. D. G. 2017, ArXiv e-prints, arXiv:1712.09357

Chen, C. H. K., Mallet, A., Schekochihin, A. A., et al. 2012,

ApJ, 758, 120

Del Zanna, L. 2001, Geophys. Res. Lett., 28, 2585

Del Zanna, L., Matteini, L., Landi, S., Verdini, A., \& Velli,

M. 2015, Journal of Plasma Physics, 81, 325810102

Del Zanna, L., Velli, M., \& Londrillo, P. 2001, A\&A, 367, 705

Derby, Jr., N. F. 1978, ApJ, 224, 1013

Goldstein, M. L. 1978, ApJ, 219, 700

Hollweg, J. V. 1994, J. Geophys. Res., 99, 23
Horbury, T. S., Forman, M., \& Oughton, S. 2008, Physical Review Letters, 101, 175005

Howes, G. G., Bale, S. D., Klein, K. G., et al. 2012, ApJL, 753, L19

Jayanti, V., \& Hollweg, J. V. 1993, J. Geophys. Res., 98, 19

Kauffmann, K., \& Araneda, J. A. 2008, Physics of Plasmas, 15, 062106

Kellogg, P. J., \& Horbury, T. S. 2005, Annales

Geophysicae, 23, 3765

Klein, K. G., Howes, G. G., TenBarge, J. M., et al. 2012, ApJ, 755, 159

Lepping, R. P., Acũna, M. H., Burlaga, L. F., et al. 1995, SSRv, 71, 207

Lin, R. P., Anderson, K. A., Ashford, S., et al. 1995, SSRv, 71,125

Malara, F., Primavera, L., \& Veltri, P. 2001, Nonlinear Processes in Geophysics, 8, 159 
Matteini, L., Landi, S., Del Zanna, L., Velli, M., \& Hellinger, P. 2010, Geophys. Res. Lett., 37, L20101

Narita, Y., \& Marsch, E. 2015, ApJ, 805, 24

Ogilvie, K. W., Chornay, D. J., Fritzenreiter, R. J., et al. 1995, SSRv, 71, 55

Pulupa, M. P., Bale, S. D., Salem, C., \& Horaites, K. 2014, Journal of Geophysical Research: Space Physics, 119, 647

Schekochihin, A. A., Cowley, S. C., Dorland, W., et al. 2009, ApJS, 182, 310

Shi, M., Li, H., Xiao, C., \& Wang, X. 2017, ApJ, 842, 63

Tenerani, A., \& Velli, M. 2013, Journal of Geophysical Research (Space Physics), 118, 7507
Tenerani, A., Velli, M., \& Hellinger, P. 2017, ApJ, 851, 99

Tu, C. Y., \& Marsch, E. 1994, Journal of Geophysical Research: Space Physics, 99, 21481

Verscharen, D., Chen, C. H. K., \& Wicks, R. T. 2017, ApJ, 840,106

Yao, S., He, J.-S., Marsch, E., et al. 2011, ApJ, 728, 146

Yao, S., He, J.-S., Tu, C.-Y., Wang, L.-H., \& Marsch, E. 2013a, ApJ, 776, 94

—. 2013b, ApJ, 774, 59 